\title{
Traveling Wave Solutions of the Extended Calogero-Bogoyavlenskii-Schiff Equation
}

\author{
S. M. Mabrouk \\ Assistant Professor, Department of physics and Engineering Mathematics \\ Faculty of Engineering, Zagazig University, Egypt.
}

\begin{abstract}
Traveling wave solutions of the extended Calogero-Bogoyavlenskii-Schiff equation (ECBS) is investigated. The $\left(G^{\prime} / G\right)$-expansion method is applied to find the explicit solutions. New traveling wave solutions of the ECBS equation are obtained. The solitary wave solutions show one-soliton, $\mathrm{N}$-solitons, kink and periodic waves. Some of the resulting solutions are plotted.
\end{abstract}

Keywords- Extended Calogero-Bogoyavlenskii-Schiff equation; $\left(G^{\prime} / G\right)$-expansion method; Traveling wave solutions.

\section{INTRODUCTION}

Nonlinear integrable equations are playing a substantial role in modeling most of the scientific and engineering applications [1-5], such as propagation of shallow water waves, optical fibers, condensed matter, electromagnetic, plasma and fluid mechanics. The study of higher dimensional nonlinear integrable equations is one of the most important recent studies [6-8]. Large assortments of mathematical methods of solutions are demoralized in studying these equations, some of these methods are the $\left(G^{\prime} / G\right)$-expansion method [9-11], exponential function method [12, 13], Lax pairs [14, 15], Extended homoclinic test [16, 17], Hirota bilinear method [18, 19], Darboux transformation [20,21], sine-cosine and tanh-coth methods [22].

Bogoyavlenskii and Schiff described the interaction of a Riemann waves along two spatial dimensions, by the nonlinear integrable equation Calogero-BogoyavlenskiiSchiff (GBS) equation [23, 24]. Riemann waves' dynamics is one of the most important applications of physics and engineering; such as tsunami and tidal in rivers, magentosound waves in plasmas, internal waves in oceans and optical tsunami in fibers.

Song et al. [25], introduced an integrable $(2+1)$ dimensional equation. This equation describes the interaction between Riemann wave promulgated along the $\mathrm{y}$-axis and long wave promulgated along the $\mathrm{x}$-axis as;

$u_{t x}+u_{x} u_{x y}+\frac{1}{2} u_{x x} u_{y}+\frac{1}{4} u_{x x x y}+\frac{1}{4} \partial_{x}^{-1} u_{y y y}=0$

Where $u(x, y, t)$ is a function of space variables $x, y$ and temporal variable t. Equation (1) is also obtained by unifying two directional generalization of the potential $\mathrm{KdV}$ equation and Calogero-Bogoyavlenskii-Shiff equation [2628]. The explicit N-soliton solutions of equation (1) is obtained in [25]. Wazwaz [18], extended equation (1) by adding the planar flux term $\beta u_{x y}$, introducing the ECBS equation; $u_{t x}+u_{x} u_{x y}+\frac{1}{2} u_{x x} u_{y}+\frac{1}{4} u_{x x x y}+\frac{1}{4} \partial_{x}^{-1} u_{y y y}+\beta u_{x y}=0$

He in [18], showed that the extension term does not disturb the integrability of the Song's equation (1), and found its Nsoliton solutions using the simplified Hirota's method.

The purpose of this paper is to find the traveling wave solutions of the extended Calogero-Bogoyavlenskii-Schiff equation (2), using the $\left(G^{\prime} / G\right)$ expansion method. The paper is organized as follows: Section II, is devoted to summarize the $\left(G^{\prime} / G\right)$ expansion method. In section III, the $\left(G^{\prime} / G\right)$ expansion method is applied to the extended Calogero-Bogoyavlenskii-Schiff equation. Section IV, is devoted to discuss the results. The paper ends with conclusions in Section V.

\section{MATHEMATICAL METHOD}

$\left(G^{\prime} / G\right)$-expansion method is excessively used in finding the traveling wave solutions of nonlinear evolution equations [29-32]. It can be summarized as; for any partial differential equation (PDE);

$P\left(u, u_{x}, u_{y}, u_{t}, u_{x x}, u_{x y}, \ldots\right)=0$

(3)

Where $p$ is a polynomial in $u$ and its partial derivatives. Suppose the solution of the partial differential equation (2.1) is in the form;

$u(x, y, t)=u(\eta), \quad \eta=x+y \pm c t$.

The constant $c$ is the velocity of the travelling wave. The PDE (3) is reduced to a nonlinear ODE; which can be integrated many times with setting the constants of integration equal to zero for simplicity. Consider the traveling wave solution of the final ODE in the form;

$u(\eta)=\sum_{i=0}^{m} a_{i}\left(\frac{G \prime}{G}\right)^{i}$

Where $G=G(\eta)$ satisfies the second order linear ODE;

$G^{\prime \prime}(\eta)+\lambda G^{\prime}(\eta)+\mu G(\eta)=0$

Where, $G^{\prime}=\frac{d G}{d \eta}, G^{\prime \prime}=\frac{d^{2} G}{d \eta^{2}}, a_{i}, \lambda$ and $\mu$ are real constants to be determined.

The positive integer $m$ is determined through balancing the highest order linear and nonlinear terms' derivatives appearing in the ODE. Substitute (5) and (6) into the final ODE, then collect all terms with the same order of $\left(G^{\prime} / G\right)$ and set each coefficient to zero yield a set of algebraic equations for $a_{i}, c, \mu$ and $\lambda$. 


\section{TRAVELING WAVE SOLUTION OF THE ECBS EQUATION}

This section presents, the application of $\left(G^{\prime} / G\right)$ expansion method to find the explicit solutions of the extended Calogero-Bogoyavlenskii-Schiff equation (2). Differentiate equation (2) with respect to $\mathrm{x}$, yields;

$4 u_{t x x}+6 u_{x x} u_{x y}+4 u_{x} u_{x x y}+2 u_{x x x} u_{y}+u_{x x x x y}+$

$u_{y y y}+4 \beta u_{x x y}=0$

We now utilize the wave transformation equation (4) in reducing (7) to the nonlinear ODE;

$(4 \beta-4 c+1) u^{\prime \prime \prime}+6\left(u^{\prime \prime}\right)^{2}+6 u^{\prime} u^{\prime \prime \prime}+u^{(5)}=0$

Where dashes refer to the derivatives with respect to $\eta$. Let

$(4 \beta-4 c+1)=\delta$

Then integrate (8) twice with respect to $\eta$ yields;

$u^{\prime \prime \prime}+3 u^{\prime 2}+\delta u^{\prime}=0$

Let $u^{\prime}=v$, yields

$v^{\prime \prime}+\delta v+3 v^{2}=0$

The balance between $v^{\prime \prime}$ and $v^{2}$, leads to $m=2$, and the solution of equation (11) is written as;

$v(\eta)=a_{0}+a_{1}\left(\frac{G \prime}{G}\right)+a_{2}\left(\frac{G^{\prime}}{G}\right)^{2}$

Where $G=G(\eta)$ satisfies equation (6) and $a_{i}, \lambda$ and $\mu$, are real constants to be determined. Substitute from (12) using (6) into (11) yields;

$\left(6 a_{2}+3 a_{2}^{2}\right)\left(\frac{G^{\prime}}{G}\right)^{4}+\left(10 a_{2} \lambda+6 a_{1} a_{2}+2 a_{1}\right)\left(\frac{G^{\prime}}{G}\right)^{3}+$

$\left(3 a_{1} \lambda+4 a_{2} \lambda^{2}+8 a_{2} \mu+\delta a_{2}+3 a_{1}^{2}+6 a_{0} a_{2}\right)\left(\frac{G^{\prime}}{G}\right)^{2}+$

$\left(a_{1} \lambda^{2}+2 a_{1} \mu+6 a_{2} \lambda \mu+\delta a_{1}+6 a_{0} a_{1}\right)\left(\frac{G^{\prime}}{G}\right)+\left(a_{1} \lambda \mu+\right.$

$\left.2 a_{2} \mu^{2}+\delta a_{0}+3 a_{0}^{2}\right)=0$

Collecting all terms with the same order of $\left(G^{\prime} / G\right)$, and setting each coefficient to zero yielding; a set of algebraic equations for $a_{i}, \delta, \mu$ and $\lambda$,

$$
\left\{\begin{array}{c}
6 a_{2}+3 a_{2}^{2}=0 \\
10 a_{2} \lambda+6 a_{1} a_{2}+2 a_{1}=0 \\
3 a_{1} \lambda+4 a_{2} \lambda^{2}+8 a_{2} \mu+\delta a_{2}+3 a_{1}^{2}+6 a_{0} a_{2}=0 \\
a_{1} \lambda^{2}+2 a_{1} \mu+6 a_{2} \lambda \mu+\delta a_{1}+6 a_{0} a_{1}=0 \\
a_{1} \lambda \mu+2 a_{2} \mu^{2}+\delta a_{0}+3 a_{0}^{2}=0
\end{array}\right.
$$

Solutions of this system of equations result in two cases;

A. Case 1; $a_{0}=-2 \mu, a_{1}=-2 \lambda, a_{2}=-2$ and $\delta=4 \mu-\lambda^{2}$

The function $G(\eta)$ is found through the solution of equation (6) by setting, $\lambda^{2}-4 \mu=\alpha$

1) The solution (12) For $\alpha>0$ is;

$v=-2 \mu-2 \lambda\left[\frac{-\lambda}{2}+\frac{\sqrt{\alpha}}{2}\left(\frac{C_{1} \sinh \left(\frac{\sqrt{\alpha}}{2} \eta\right)+C_{2} \cosh \left(\frac{\sqrt{\alpha}}{2} \eta\right)}{C_{1} \cosh \left(\frac{\sqrt{\alpha}}{2} \eta\right)+C_{2} \sinh \left(\frac{\sqrt{\alpha}}{2} \eta\right)}\right)\right]-$

$2\left[\frac{-\lambda}{2}+\frac{\sqrt{\alpha}}{2}\left(\frac{C_{1} \sinh \left(\frac{\sqrt{\alpha}}{2} \eta\right)+C_{2} \cosh \left(\frac{\sqrt{\alpha}}{2} \eta\right)}{C_{1} \cosh \left(\frac{\sqrt{\alpha}}{2} \eta\right)+C_{2} \sinh \left(\frac{\sqrt{\alpha}}{2} \eta\right)}\right)\right]^{2}$

Which can be simplified for $C_{1}=0$ and $C_{2}=1$, to;

$v_{1}=-2 \mu+\frac{\lambda^{2}}{2}-\frac{\alpha}{2} \operatorname{coth}^{2}\left(\frac{\sqrt{\alpha}}{2} \eta\right)$ $v_{2}=-2 \mu+\frac{\lambda^{2}}{2}-\frac{\alpha}{2} \tanh ^{2}\left(\frac{\sqrt{\alpha}}{2} \eta\right)$

2) Equation (12) for $<0$, is;

$v=-2 \mu-2 \lambda\left[\frac{-\lambda}{2}+\frac{\sqrt{-\alpha}}{2}\left(\frac{C_{1} \sin \left(\frac{\sqrt{-\alpha}}{2} \eta\right)+C_{2} \cos \left(\frac{\sqrt{-\alpha}}{2} \eta\right)}{C_{1} \cos \left(\frac{\sqrt{-\alpha}}{2} \eta\right)+C_{2} \sin \left(\frac{\sqrt{-\alpha}}{2} \eta\right)}\right)\right]-$

$2\left[\frac{-\lambda}{2}+\frac{\sqrt{-\alpha}}{2}\left(\frac{C_{1} \sin \left(\frac{\sqrt{-\alpha}}{2} \eta\right)+C_{2} \cos \left(\frac{\sqrt{-\alpha}}{2} \eta\right)}{C_{1} \cos \left(\frac{\sqrt{-\alpha}}{2} \eta\right)+C_{2} \sin \left(\frac{\sqrt{-\alpha}}{2} \eta\right)}\right)\right]^{2}$

Which can be simplified for $C_{1}=0$ and $C_{2}=1$, to;

$v_{3}=-2 \mu+\frac{\lambda^{2}}{2}-\frac{\alpha}{2} \cot ^{2}\left(\frac{\sqrt{-\alpha}}{2} \eta\right)$

And for $C_{1}=1$ and $C_{2}=0$, to;

$v_{4}=-2 \mu+\frac{\lambda^{2}}{2}+\frac{\alpha}{2} \tan ^{2}\left(\frac{\sqrt{-\alpha}}{2} \xi\right)$

B. Case 2; $a_{0}=-\frac{\lambda^{2}}{3}-\frac{2}{3} \mu, a_{1}=-2 \lambda, a_{2}=-2$ and $\delta=\lambda^{2}-4 \mu$

1) For $\alpha>0$, equation (12) becomes;

$v=-\frac{\lambda^{2}}{3}-\frac{2}{3} \mu-2 \lambda\left[\frac{-\lambda}{2}+\right.$

$\left.\frac{\sqrt{\alpha}}{2}\left(\frac{C_{1} \sinh \left(\frac{\sqrt{\alpha}}{2} \eta\right)+C_{2} \cosh \left(\frac{\sqrt{\alpha}}{2} \eta\right)}{C_{1} \cosh \left(\frac{\sqrt{\alpha}}{2} \eta\right)+C_{2} \sinh \left(\frac{\sqrt{\alpha}}{2} \eta\right)}\right)\right]-2\left[\frac{-\lambda}{2}+\right.$

$\left.\frac{\sqrt{\alpha}}{2}\left(\frac{C_{1} \sinh \left(\frac{\sqrt{\alpha}}{2} \eta\right)+C_{2} \cosh \left(\frac{\sqrt{\alpha}}{2} \eta\right)}{C_{1} \cosh \left(\frac{\sqrt{\alpha}}{2} \eta\right)+C_{2} \sinh \left(\frac{\sqrt{\alpha}}{2} \eta\right)}\right)\right]^{2}$

at $C_{1}=0$ and $C_{2}=1$, we have;

$v_{5}=\frac{\lambda^{2}}{6}-\frac{2}{3} \mu-\frac{\alpha}{2} \operatorname{coth}^{2}\left(\frac{\sqrt{\alpha}}{2} \eta\right)$

And at $C_{1}=1$ and $C_{2}=0$, we have;

$v_{6}=\frac{\lambda^{2}}{6}-\frac{2}{3} \mu-\frac{\alpha}{2} \tanh ^{2}\left(\frac{\sqrt{\alpha}}{2} \eta\right)$

2) For $\alpha<0$, equation (12) becomes;

$v=-\frac{\lambda^{2}}{3}-\frac{2}{3} \mu-2 \lambda\left[\frac{-\lambda}{2}+\right.$

$\left.\frac{\sqrt{-\alpha}}{2}\left(\frac{C_{1} \sin \left(\frac{\sqrt{-\alpha}}{2} \eta\right)+C_{2} \cos \left(\frac{\sqrt{-\alpha}}{2} \eta\right)}{C_{1} \cos \left(\frac{\sqrt{-\alpha}}{2} \eta\right)+C_{2} \sin \left(\frac{\sqrt{-\alpha}}{2} \eta\right)}\right)\right]-2\left[\frac{-\lambda}{2}+\right.$

$\left.\frac{\sqrt{-\alpha}}{2}\left(\frac{C_{1} \sin \left(\frac{\sqrt{-\alpha}}{2} \eta\right)+C_{2} \cos \left(\frac{\sqrt{-\alpha}}{2} \eta\right)}{C_{1} \cos \left(\frac{\sqrt{-\alpha}}{2} \eta\right)+C_{2} \sin \left(\frac{\sqrt{-\alpha}}{2} \eta\right)}\right)\right]^{2}$

$C_{1}=0$ and $C_{2}=1$, yields;

$v_{7}=\frac{\lambda^{2}}{6}-\frac{2}{3} \mu+\frac{\alpha}{2} \cot ^{2}\left(\frac{\sqrt{-\alpha}}{2} \eta\right)$

$C_{1}=1$ and $C_{2}=0$, yields;

$v_{8}=\frac{\lambda^{2}}{6}-\frac{2}{3} \mu+\frac{\alpha}{2} \tan ^{2}\left(\frac{\sqrt{-\alpha}}{2} \eta\right)$

\section{RESULTS AND DISCUSSION}

This section is motivated to identify and plot the traveling wave solutions of ECBS equation (2), for the two cases;

\section{A. Traveling wave solutions for case 1}

Integrating equation (16) with respect to $\eta$ yields, a onesoliton solution $u_{1}$ of the ECBS equation;

$u_{1}=\sqrt{\alpha} \operatorname{coth}\left(\frac{\sqrt{\alpha}}{2} \eta\right)=\sqrt{\alpha} \operatorname{coth}\left(\frac{\sqrt{\alpha}}{2}(x+y+\delta t)\right)$

This solution is plotted in Fig. 1 for $\alpha=2, t=2$ and $\delta=-1$. 


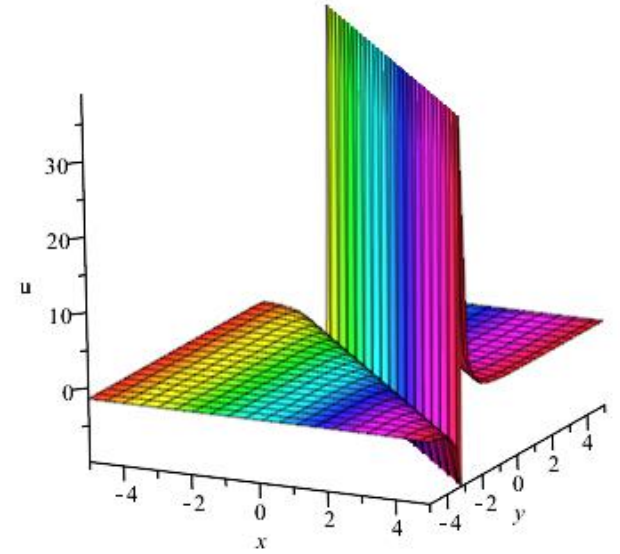

Fig.1. One soliton solution of EBCS equation for $\alpha=2, t=2$ and $\delta=-1$

Integrating (17) with respect to $\eta$ yields, a kink-soliton solution $u_{2}$ of the ECBS equation, as shown in Fig.2, for $\alpha$ $=2, t=0.5$ and $\delta=-2$.

$u_{2}=\sqrt{\alpha} \tanh \left(\frac{\sqrt{\alpha}}{2} \eta\right)=\sqrt{\alpha} \tanh \left(\frac{\sqrt{\alpha}}{2}(x+y+\delta t)\right)$

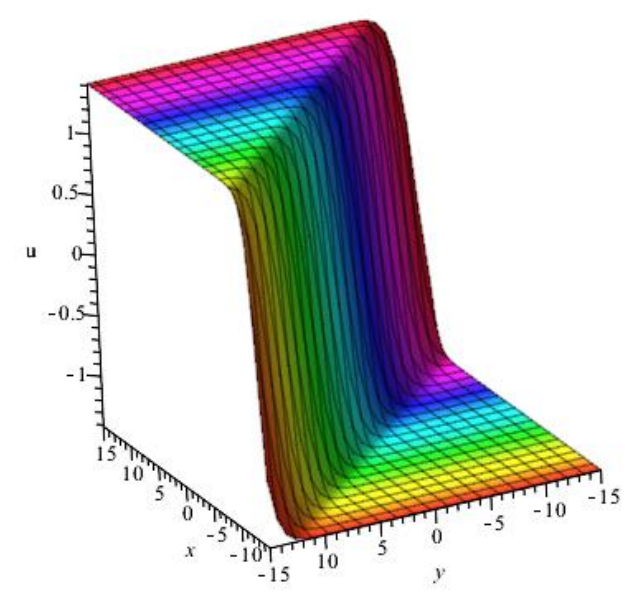

Fig.2. Kink solution of ECBS equation for $\delta=2, t=0.5$ and $\delta=-2$.

Integrating (19) with respect to $\eta$ yields, a periodic solution $u_{3}$ of the ECBS equation, as presented in Fig.3, for $\alpha=2, t$ $=1$ and $\delta=1$.

$u_{3}=\sqrt{-\alpha} \cot \left(\frac{\sqrt{-\alpha}}{2} \eta\right)=\sqrt{-\alpha} \cot \left(\frac{\sqrt{-\alpha}}{2}(x+y+\delta t)\right)$

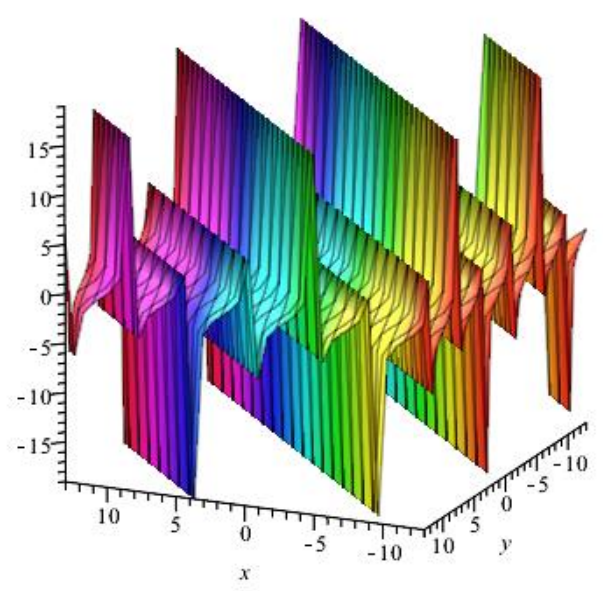

Fig.3. Periodic solution of ECBS equation for $\alpha=2, t=1$ and $\delta=1$.

Integrating (20) with respect to $\eta$ yields, an N-soliton solution $u_{4}$ of the ECBS equation, as presented in Fig.4, for $\alpha=2, t=0.2$ and $\delta=1$.

$$
u_{4}=-\sqrt{-\alpha} \tan \left(\frac{\sqrt{-\alpha}}{2} \eta\right)=-\sqrt{-\alpha} \tan \left(\frac{\sqrt{-\alpha}}{2}(x+y+\delta t)\right)
$$

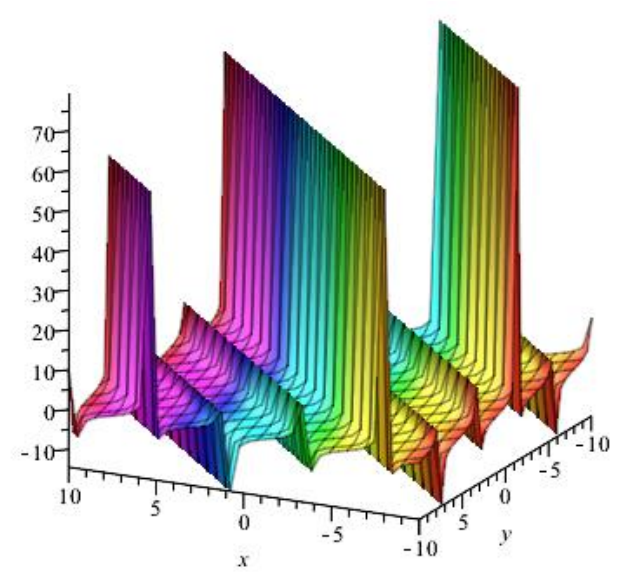

Fig.4. N-soliton solution of ECBS equation for $\alpha=2, \mathrm{t}=0.2$ and $\delta=1$.

\section{B. Traveling wave solutions for case 2}

Integrating (22) with respect to $\eta$ yields, a one-soliton solution $u_{5}$ of the ECBS equation;

$u_{5}=-\frac{\alpha}{3} \eta+\sqrt{\alpha} \operatorname{coth}\left(\frac{\sqrt{\alpha}}{2} \eta\right)$

Integrating (23) with respect to $\eta$ yields;

$u_{6}=-\frac{\alpha}{3} \eta+\sqrt{\alpha} \tanh \left(\frac{\sqrt{\alpha}}{2} \eta\right)$

Integrating (25) with respect to $\eta$ yields,

$u_{7}=-\frac{\alpha}{3} \eta+\sqrt{-\alpha} \cot \left(\frac{\sqrt{-\alpha}}{2} \eta\right)$

Integrating (26) with respect to $\eta$ yields,

$u_{8}=-\frac{\alpha}{3} \xi-\sqrt{-\alpha} \tan \left(\frac{\sqrt{-\alpha}}{2} \eta\right)$ 


\section{CONCLUSIONS}

In this paper, the $\left(G^{\prime} / G\right)$-expansion method is effectively employed to reveal many explicit solutions for the extended Calogero-Bogoyavlenskii-Schiff equation. The presented solutions include a variety of one-soliton, kink, periodic and $\mathrm{N}$-soliton solutions. These aggregations of traveling wave solutions are applicable for different conditions. The results help in studying the wave behaviors in several applications as, deep oceans and nonlinear optics.

\section{REFERENCES}

[1] M. Veera Krishna, G. Subba Reddy, MHD Forced Convective flow of Non-Newtonian fluid through Stumpy Permeable Porous medium, Materials Today: Proceedings: Part 1, 5 (2018) 175-183.

[2] A.S. Rashed, Analysis of $(3+1)$-dimensional unsteady gas flow using optimal system of Lie symmetries, Mathematics and Computers in Simulation, 156 (2019) 327-346.

[3] Y. Wang, X. Li, Entire solutions for the classical competitive LotkaVolterra system with diffusion in the weak competition case, Nonlinear Analysis: Real World Applications, 42 (2018) 1-23.

[4] Y. Tian, I. Al-Darabsah, Y. Yuan, Global dynamics in sea lice model with stage structure, Nonlinear analysis.Real world applications, 44 (2018) 283-304

[5] S.M. Mabrouk, A.S. Rashed, N-Solitons, kink and periodic wave solutions for $(3+1)$-dimensional Hirota bilinear equation using three distinct techniques, Chinese Journal of Physics, 60 (2019) 4860.

[6] R. Saleh, S.M. Mabrouk, M. Kassem, Truncation method with point transformation for exact solution of Liouville Bratu Gelfand equation, Computers and Mathematics with Applications, 76 (2018) 1219-1227.

[7] S.M. Mabrouk, A.S. Rashed, Analysis of (3+1)-dimensional BoitiLeon-Manna-Pempinelli equation via Lax pair investigation and group transformation method, Computers and Mathematics with Applications, 74 (2017) 2546-2556.

[8] M.O. Al-Amr, S. El-Ganaini, New exact traveling wave solutions of the (4+1)-dimensional Fokas equation, Computers \& Mathematics with Applications, 74 (2017) 1274-1287.

[9] M. Abdollahzadeh, M. Ghanbarpour, A. Hosseini, S. Kashani, Exact travelling solutions for Benjamin-Bona-Mahony-Burgers equations by $\left(\mathrm{G}^{\prime} / \mathrm{G}\right)$-expansion method, International Journal of Applied Mathematics and Computation, 3 (2010) 70-76.

[10] M.A. Akbar, N.H.M. Ali, F.A. Abdullah, The Generalized and Improved ( $\left.\mathrm{G}^{\prime} / \mathrm{G}\right)$-Expansion Method with the Jacobi Elliptic Equation for Exact Solutions of Nonlinear Evolution Equations, World Applied Sciences Journal, 23 (2013) 279-288.

[11] C. Teymuri Sindi, J. Manafian, Wave solutions for variants of the $\mathrm{KdV}-$ Burger and the $\mathrm{K}(\mathrm{n}, \mathrm{n})$-Burger equations by the generalized $\mathrm{G}^{\prime} / \mathrm{G}$-expansion method, Mathematical Methods in the Applied Sciences, 40 (2017) 4350-4363.

[12] M. Abdelrahman, E. Zahran, M. Khater, The exp- ( $-\phi(\xi))-$ Expansion Method and Its Application for Solving Nonlinear Evolution Equations, International Journal of Modern Nonlinear Theory and Application, 4 (2015) 37-47.

[13] H.-O. Roshid, M. Azizur Rahman, The $\exp (-\Phi(\eta))$-expansion method with application in the $(1+1)$-dimensional classical Boussinesq equations, Results in Physics, 4 (2014) 150-155.

[14] Z.-Z. Lan, Y.-T. Gao, J.-W. Yang, C.-Q. Su, B.-Q. Mao, Solitons, Bäcklund transformation and Lax pair for a $(2+1)$-dimensional Broer-Kaup-Kupershmidt system in the shallow water of uniform depth, Communications in Nonlinear Science and Numerical Simulation, 44 (2017) 360-372.

[15] D.W. Zuo, Y.T. Gao, L. Xue, Y.J. Feng, Lax pair, rogue-wave and soliton solutions for a variable-coefficient generalized nonlinear Schrödinger equation in an optical fiber, fluid or plasma, Optical and Quantum Electronics, 48 (2016) 76-90

[16] Z. Dai, J. Liu, D. Li, Applications of HTA and EHTA to YTSF Equation, Applied Mathematics and Computation, 207 (2009) 360364.
[17] M. Najafi, M. Najafi, M.T. Darvishi, New Exact Solutions to the (2+1)-Dimensional Ablowitz-Kaup-Newell-Segur Equation: Modification of the Extended Homoclinic Test Approach, Chinese Physics Letters, 29 (2012) 40202

[18] A.M. Wazwaz, A study on two extensions of the BogoyavlenskiiSchieff equation, Communications in Nonlinear Science and Numerical Simulation, 17 (2012) 1500-1505.

[19] A.M. Wazwaz, S.A. El Tantawy, Solving the $(3+1)$-dimensional KP-Boussinesq and BKP-Boussinesq equations by the simplified Hirota's method, Nonlinear Dynamics, 88 (2017) 3017-3021.

[20] Z.X. Zhou, Darboux transformations and global solutions for a nonlocal derivative nonlinear Schrödinger equation, Communications in Nonlinear Science and Numerical Simulation, 62 (2018) 480-488

[21] L.Y. Ma, H.Q. Zhao, S.F. Shen, W.X. Ma, Abundant exact solutions to the discrete complex mKdV equation by Darboux transformation, Communications in Nonlinear Science and Numerical Simulation, 68 (2019) 31-40

[22] A.M. Wazwaz, The sine-cosine and the tanh methods: Reliable tools for analytic treatment of nonlinear dispersive equations, Applied Mathematics and Computation, 173 (2006) 150-164.

[23] O.I. Bogoyavlenskiı̌, OVAB - Two two-dimensional nonlinear equations are constructed which are integrable by means of a onedimensional inverse scattering problem. Soliton and N-soliton solutions are indicated which are smooth in one coordinate and in the other possess the same overturning property as the classical Riemann wave. ERTURNING SOLITONS IN NEW TWODIMENSIONAL INTEGRABLE EQUATIONS, Mathematics of the USSR-Izvestiya, 34 (1990) 245-259.

[24] O.I. Bogoyavlenskii, Breaking solitons in 2+1-dimensiona integrable equations, Russian Mathematical Surveys, 45 (1990) 1 86.

[25] S. Yu, K. Toda, T. Fukuyama, N-Soliton Solutions to a New $(2+1)$ Dimensional Integrable Equation, 1998

[26] S. Li, Y. Li, B.g. Zhang, Some singular solutions and their limit forms for generalized Calogero-Bogoyavlenskii-Schiff equation, An International Journal of Nonlinear Dynamics and Chaos in Engineering Systems, 85 (2016) 1665-1677.

[27] S. San, A. Akbulut, Ö. Unsal, F. Tascan, Conservation laws and double reduction of $(2+1)$ dimensional Calogero-BogoyavlenskiiSchiff equation, Mathematical Methods in the Applied Sciences, 40 (2017) 1703-1710

[28] K. Ayub, M.Y. Khan, Q. Mahmood-Ul-Hassan, Solitary and periodic wave solutions of Calogero-Bogoyavlenskii-Schiff equation via exp-function methods, Computers \& mathematics with applications, 74 (2017) 3231-3241.

[29] M. Wang, X. Li, J. Zhang, The ( G'/ G )-expansion method and travelling wave solutions of nonlinear evolution equations in mathematical physics, Physics Letters A, 372 (2008) 417-423.

[30] E. Parkes, Observations on the basic $\left(\mathrm{G}^{\prime} / \mathrm{G}\right)$-expansion method for finding solutions to nonlinear evolution equations, Applied Mathematics and Computation 217 (2010).

[31] M.A. Akbar, N.H.M. Ali, E.M.E Zayed, A Generalized and Improved (G'/G)-Expansion Method for Nonlinear Evolution Equations, Mathematical Problems in Engineering, 2012 (2012) 22.

[32] M.A. Akbar, N.H.M. Ali, E.M.E. Zayed, Abundant exact traveling wave solutions of generalized Bretherton equation via improved (G'/G)-expansion method, Communications in Theoretical Physics, 57 (2012) 173-178. 